\title{
Anaphylaxis during intraoperative indocyanine green angiography: A complication to watch out
}

\author{
Harnarayan Singh, Ankur Bajaj, Kiran Jangra1, Sviashanmugam Dhandapani
}

\begin{abstract}
Indocyanine green (ICG) angiography is widely used in cerebrovascular neurosurgery. We would like to report a case of intraoperative anaphylaxis to ICG during aneurysmal clipping and a view from the neurosurgeons' perspective during such an event.
\end{abstract}

Key words: Anaphylaxis, indocyanine green, neurosurgery

\section{INTRODUCTION}

Indocyanine green (ICG) angiography exists since 2003 in the field of cerebrovascular surgery. The popularity of ICG is gaining ground in the neurosurgical speciality due to the competition offered by endovascular embolisation in terms of not compromising the main vessels not jeopardising the safety of perforators along with satisfactory obliteration of an aneurysm. After achieving satisfactory results with vascular microsurgery, new avenues are being considered to obtain safety of the vessels around, along with complete obliteration of aneurysm. Intraoperative digital substraction angiography (Op-DSA) is increasingly being used in advanced care centres to ascertain the perfection in clipping technique. ICG being a simpler, non-invasive, no requirement of additional facility and expertise perhaps may be a more acceptable modality with efficacy close to Op-DSA. However, unlike their counterparts in

Departments of Neurosurgery and ${ }^{1}$ Anaesthesia and Intensive Care, Postgraduate Institute of Medical Education and Research, Chandigarh, India

\section{Address for correspondence:}

Dr. Sviashanmugam Dhandapani, Department of Neurosurgery,

Postgraduate Institute of Medical Education and Research, Chandigarh, India.

E-mail: ssdhandapani.neurosurg@gmail.com

\begin{tabular}{|l|l|}
\hline \multicolumn{2}{|c|}{ Access this article online } \\
\hline Quick Response Code: & Website: \\
\hline & www.jnaccjournal.org \\
\cline { 2 - 3 } & \\
\hline
\end{tabular}

ophthalmology and cardiology who have been using ICG for a fairly long time, neurosurgeons are just exploring the possibilities of its usefulness. There is no doubt of it being a surgeon and patient friendly modality. We would like to report a case of anaphylaxis to ICG during aneurysmal surgery. This is indeed a rare occurrence; however, it is always prudent to be extra cautious.

\section{CASE REPORT}

A 55-year-old female presented with sudden severe headache and vomiting. She was a known case of hypertension for 10 years, for which she was on medication amlodipine, $5 \mathrm{mg}$ OD. She was conscious, alert and with no focal deficits (Hunt and Hess Grade II). The patient had no previous history of allergy to iodides. Non-contrast computed tomography (CT) head showed a bleed in the anterior interhemispheric fissure, a haematoma in the genu of corpus callosum and cingulate gyrus. The patient underwent CT angiography, which confirmed an aneurysm arising from the bifurcation site of an azygos A2 segment of the anterior cerebral artery. The patient was taken up for bifrontal craniotomy and clipping of the aneurysm. Baseline blood pressure (BP) was $124 / 88 \mathrm{mmHg}$, and pulse rate (PR) was $81 / \mathrm{min}$. Anaesthetic induction was performed with intravenous

This is an open access article distributed under the terms of the Creative Commons Attribution-NonCommercial-ShareAlike 3.0 License, which allows others to remix, tweak, and build upon the work non-commercially, as long as the author is credited and the new creations are licensed under the identical terms.

For reprints contact: reprints@medknow.com

How to cite this article: Singh $\mathrm{H}$, Bajaj A, Jangra K, Dhandapani S. Anaphylaxis during intraoperative indocyanine green angiography: A complication to watch out. J Neuroanaesthesiol Crit Care 2016;3:126-8. 
propofol and vecuronium bolus. Propofol infusion was used to maintain adequated depth of anaesthesia. Intra-operatively, ICG angiography was done before clipping the aneurysm $(10 \mathrm{mg}, 0.05 \%)$ to define the disposition of vessels as it was not clear due to thick clot. After the first dose of ICG, BP went down to $100 / 70 \mathrm{mmHg}$, and $\mathrm{PR}$ was $85 / \mathrm{min}$. $\mathrm{BP}$ responded to fluid infusion and returned back to baseline after $10 \mathrm{~min}$ of the first injection. After clipping the aneurysm, there was doubt of callosomarginal branch being compromised by the clip, so it was repeated with $10 \mathrm{mg} 15 \mathrm{~min}$ following the first dose of ICG. Clip was modified so that all vessels had normal filling. After second ICG injection, patient suddenly developed profound hypotension (72/40 $\mathrm{mmHg}$ ) with tachycardia (PR - 118/min) and increase in airway pressure up to $35 \mathrm{~cm}$ of $\mathrm{H}_{2} \mathrm{O}$. The patient responded poorly to fluids and dopamine infusion. There was no evidence of sudden blood loss, excessive depth of anaesthesia or use of papaverine by the surgeon. At this point, anaphylaxis was suspected. Adrenaline $10 \mu \mathrm{g}$ bolus was given followed by infusion to maintain haemodynamics. The patient also received pheniramine maleate, hydrocortisone and ranitidine. Thereafter, patients' vitals started normalising and returned to baseline within $2 \mathrm{~h}$. Both the inotropes were stopped by the end of surgery.

The brain also started bulging during this time. There was no operative site haematoma, and ventricular tap revealed minimal volume of cerebrospinal fluid under low pressure. Hence, a loose duraplasty was done, and the bone flap was placed in a subcutaneous pocket in the thigh. Post-operative CT revealed full brain with small ventricles. The patient gradually stabilised over a period of 2 days. In the post-operative period, the patient developed right lower limb weakness, which was attributed to vasospasm. Vasospasm was diagnosed based on clinical symptoms, transcranial doppler and DSA to confirm the diagnosis. Vasospasm did not respond to triple-H therapy. She underwent DSA and intra-arterial nimodipine infusion. The patient made an excellent recovery with no motor deficits.

\section{DISCUSSION}

The management of patients with aneurysmal subarachnoid haemorrhage has undergone immense changes in the last decade. ${ }^{[1]}$ Endovascular coiling has yet to replace surgical clipping as the modality of choice in many centres. ICG angiography has become a more acceptable alternative to Op-DSA to assess the adequacy of aneurysm obliteration and patency of adjacent vessels.

ICG is a near-infrared (NIR) spectrum fluorescent dye. The absorption and emission peaks of ICG (805 and $835 \mathrm{~nm}$, respectively) lie within the 'optical window' of tissue, where absorption attributable to endogenous chromophores is low. NIR light can, therefore, penetrate tissue to depths of several millimetres to a few centimetres. After intravenous injection, ICG is bound within 1 to $2 \mathrm{~s}$, mainly to globulins ( $\alpha 1$-lipoproteins), and remains intravascular, with normal vascular permeability. ICG is not metabolised in the body and is excreted exclusively by the liver, with a plasma half-life of 3 to $4 \mathrm{~min}$. It is not reabsorbed from the intestine and does not undergo enterohepatic recirculation. ${ }^{[2]}$

ICG has been used for many years by cardiologists for cardiac output studies, and ophthalmologists for studying the choroidal vasculature. ICG angiography has recently found a niche in the field of cerebrovascular surgery. Raabe et al. ${ }^{[2]}$ have done pioneering work with this technique. The beauty of ICG angiography lies in its ability to demonstrate the patency of blood vessels during aneurysm surgery, ${ }^{[2,3]}$ confirm patency of microvascular anastomosis ${ }^{[4,5]}$ and arteriovenous shunt obliteration. ${ }^{[6]}$

ICG angiography has a high concordance rate with Op-DSA in terms of evaluation of safety during clip application. ${ }^{[7]}$ ICG angiography is relatively easier, simpler and does not require additional equipment/expertise, hence, this is more patient and surgeon friendly as compared to the 'gold standard' Op-DSA.

There is large experience with the use of ICG in the literature of cardiology and ophthalmology. Only a few case reports, however, exist in literature describing the adverse reactions to this compound, especially the life-threatening anaphylactic reactions. ${ }^{[8]}$ Intra-operatively management of anaphylaxis may be extremely difficult for both the surgeon and the anaesthesiologist during a cranial surgery. It is difficult to diagnose anaphylaxis intra-operatively. During general anaesthesia, patients may have altered sympatho-adrenergic response to acute anaphylactic shock. The sign and symptoms of anaphylactic reactions documented in case reports include urticaria, erythema, hypotension, pulmonary congestion and laryngospasm. As the patient is under drapes, and mostly unconscious or sedated, the early cutaneous signs of anaphylaxis remain often unrecognised leaving bronchospasm, and cardiovascular collapse as the first recognised signs of anaphylaxis. In our anaesthetised patient, we did not encounter any heralding cutaneous markers and rapidly developed hypotension and tachycardia.

Anaphylaxis during cranial surgery is rarely reported in the literature. The two cases reported by O'Reilly et al., ${ }^{[9]}$ from neurosurgery did not involve a craniotomy. Only one case of anaphylaxis associated with ICG 
administration during surgery for intracranial aneurysm has been reported. ${ }^{[8]}$

Anaphylaxis induced systemic disturbances can pose serious surgical challenges. Hypotension increases intracranial volume by reflex vasodilatation leading to brain bulge. Bronchospasm raises airway pressures, which further leads to worsening of the surgical field. Hypotension with brain bulge compromises cerebral perfusion. In the case reported, the aneurysm had been clipped when the reaction occurred, and, fortunately, the clip did not require readjustment. We recommend a sufficient craniotomy and duroplasty in the case of such inadvertent event, to manage raised intracranial pressure.

The applications of ICG in the field of neurosurgery can be the next turning point in the progress of cerebrovascular surgery. The vast potential is still being explored. However, one should keep eyes open for an anaphylactic reaction to this compound. We conclude that one should be extremely vigilant while injecting ICG to prevent even the mildest form of anaphylaxis.

\section{Financial support and sponsorship}

Nil.

\section{Conflicts of interest}

There are no conflicts of interest.

\section{REFERENCES}

1. Dhandapani S, Goudihalli S, Mukherjee KK, Singh H, Srinivasan A, Danish $\mathrm{M}$, et al. Prospective study of the correlation between admission plasma homocysteine levels and neurological outcome following subarachnoid hemorrhage: A case for the reverse epidemiology paradox? Acta Neurochir (Wien) 2015;157:399-407.

2. Raabe A, Beck J, Gerlach R, Zimmermann M, Seifert V. Near-infrared indocyanine green video angiography: A new method for intraoperative assessment of vascular flow. Neurosurgery 2003;52:132-9.

3. de Oliveira JG, Beck J, Seifert V, Teixeira MJ, Raabe A. Assessment of flow in perforating arteries during intracranial aneurysm surgery using intraoperative near-infrared indocyanine green video angiography. Neurosurgery 2007;61 Suppl 3:63-73.

4. Awano T, Sakatani K, Yokose N, Kondo Y, Igarashi T, Hoshino $\mathrm{T}$, et al. Intraoperative EC-IC bypass blood flow assessment with indocyanine green angiography in moyamoya and non-moyamoya ischemic stroke. World Neurosurg 2010;73:668-74.

5. Misra BK, Purandare HR, Warade AG. Indocyanine green dye available in India is good for microscope integrated near infrared video angiography. Neurol India 2011;59:321-2.

6. Killory BD, Nakaji P, Gonzales LF, Ponce FA, Wait SD, Spetzler RF. Prospective evaluation of surgical microscope-integrated intraoperative near-infrared indocyanine green angiography during cerebral arteriovenous malformation surgery. Neurosurgery 2009;65:456-62.

7. Washington CW, Zipfel GJ, Chicoine MR, Derdeyn CP, Rich KM, Moran CJ, et al. Comparing indocyanine green video angiography to the gold standard of intraoperative digital subtraction angiography used in aneurysm surgery. J Neurosurg 2013;118:420-7.

8. Welch MB, Moore LE. Anaphylaxis associated with indocyanine green administration for intraoperative fluorescence angiography. In: Mashour GA, Farag E, editors. Case Studies in Neuroanesthesia and Neurocritical Care. New York: Cambridge University Press; 2011. p. 66-9.

9. O'Reilly G, Tatman A, Hockley AD. Intraoperative anaphylaxis during neurosurgery. Childs Nerv Syst 1998;14:185-7. 